\title{
Modelación y simulación de un pervaporador acoplado a un proceso de sacarificación-fermentación para la producción de etanol
}

\author{
Modeling and simulation of a pervaporator coupled to a \\ simultaneous saccharification-fermentation process for the \\ ethanol production
}
Modelação e simulação de um pervaporador acoplado a um processo de sacarificação-fermentação para a produção de etanol

Fecha Recepción: 30 de Junio de 2015

Jairo Antonio Cubillos-Lobo*

Fecha Aprobación: 18 de Agosto de 2015

Felipe Bustamante-Londoño**

Alejandro Acosta-Cárdenas ${ }^{* * *}$

\section{Resumen}

Actualmente, la integración de procesos es considerada una opción viable para reducir costos en la producción de etanol a partir de biomasa. Simulaciones y resultados experimentales han demostrado los beneficios de la integración de las etapas de sacarificación y fermentación y del acoplamiento del proceso de fermentación a la recuperación in-situ de etanol por pervaporación en la producción de bioetanol; sin embargo, no se han publicado estudios de la integración del proceso de sacarificación-fermentación simultánea con membranas de separación, para la remoción insitu de etanol a partir del caldo de fermentación. En este trabajo se aborda esta necesidad, mediante la modelación y simulación de la producción de etanol a partir de almidón de yuca por sacarificación-fermentación simultánea, acopladas a un sistema de remoción in-situ de etanol por pervaporación, con membranas a base de polidimetilsiloxano (PDMS), silicalita y PDMS-Silicalita. La membrana de PDMS se modeló usando el mecanismo de solución-difusión, mientras que para la membrana de silicalita se utilizó el modelo de adsorción-difusión. Para el modelo del proceso de sacarificación-fermentación simultánea (SSF) se utilizó una fusión del modelo multicadena (Michaelis-Menten), junto con un modelo tipo Monod. El ajuste

* Ph. D. Universidad Pedagógica y Tecnológica de Colombia (Tunja-Boyacá, Colombia). jairo.cubillos@uptc.edu.co.

** Ph. D. Universidad de Antioquia (Medellín-Antioquia, Colombia). fbustama@udea.edu.co.

*** M. Sc. Universidad de Antioquia (Medellín-Antioquia, Colombia). alejandro.acosta@udea.edu.co. 
Modelación y simulación de un pervaporador acoplado a un proceso de sacarificación-fermentación para la producción de etanol

del modelo de SSF a los datos experimentales reportados, así como de los modelos de membranas de PDMS y silicalita a los valores reportados en la literatura es bueno: la máxima desviación encontrada es del orden de $3 \%$. El modelo integrado se utilizó para predecir la concentración de etanol en función del tiempo durante la sacarificación-fermentación simultánea.

Palabras clave: Sacarificación-fermentación simultánea, Bioetanol, Pervaporación, PDMS, Silicalita, Modelación y simulación, Integración de procesos.

\section{Abstract}

Process integration is now days considered a viable option for reducing ethanol production costs from biomass. Both experimental and simulation results have shown the benefits of coupling saccharification and fermentation as well as fermentation and pervaporation. However, the integration of simultaneous saccharificationfermentation with membrane-based in-situ removal of ethanol, which would allow reaching the benefits of this approach, has not been reported yet. This work aims to obtain the modelling and simulation's results in the production of ethanol, from cassava starch by simultaneous saccharification and fermentation, coupled with the membranes separation, based on Silicalite PDMS, and PDMS-Silicalite.

A combined solution-diffusion mechanism and adsorption-diffusion mechanism was used for modelling the PDMS membrane where as the Maxwell-Stefan multi-component mass-transfer equations were used for modelling the silicalite membrane..A simultaneous-saccharification and fermentation process was modelled, using a multi-chain model (Michaelis-Menten) coupled with the Monod model. The maximum deviation found in the adjusted SSF model to the reported experimental data, as well as the values for PDMS and silicalite membrane models, is in the 3\% range. The integrated model was used to predict the ethanol concentration during the simultaneous saccharification-fermentation process.

Keywords: Simultaneous Saccharification Fermentation, Bioethanol, Pervaporation, PDMS, Silicalite, Modelling and Simulation, Process integration.

\section{Resumo}

Atualmente, a integração de processos é considerada uma opção viável para reduzir custos na produção de etanol a partir de biomassa. Simulações e resultados experimentais têm demonstrado os benefícios da integração das etapas de sacarificação e fermentação e do acoplamento do processo de fermentação à recuperação in situ de etanol por pervaporação na produção de bioetanol; porém, não tem sido publicados estudos da integração do processo de sacarificação-fermentação simultânea com membranas de separação, para a remoção in situ de etanol a partir do caldo de fermentação. Neste trabalho se aborda esta necessidade, mediante a modelação e simulação da produção de etanol a partir de amido de mandioca por sacarificação-fermentação simultânea, acopladas a um sistema de remoção in situ de etanol por pervaporação, com membranas a base de polidimetilsiloxano (PDMS), silicalita e PDMS-Silicalita. A membrana de PDMS modelou-se usando o mecanismo de solução-difusão, enquanto que para a membrana de silicalita se utilizou o modelo de adsorção-difusão. Para o modelo do processo de sacarificaçãofermentação simultânea (SSF) utilizou-se uma fusão do modelo de várias correntes (Michaelis-Menten), junto com um modelo tipo Monod. O ajuste do modelo de SSF aos dados experimentais reportados, assim como dos modelos de membranas de PDMS e silicalita aos valores reportados na literatura é bom: o máximo desvio encontrado é da ordem de 3\%. O modelo integrado utilizou-se para predizer a concentração de etanol em função do tempo durante a sacarificação-fermentação simultânea.

Palavras chave: Sacarificação-fermentação simultânea, Bioetanol, Pervaporação, PDMS, Silicalita, Modelação e simulação, Integração de processos. 


\section{INTRODUCCIÓN}

Actualmente, el biocombustible más importante es el etanol, producto completamente renovable obtenido a partir de cultivos bioenergéticos y biomasa. El etanol es utilizado como aditivo oxigenante de la gasolina, con el fin de reducir las emisiones de monóxido de carbono, compuestos aromáticos y compuestos orgánicos volátiles [1]. Tradicionalmente, la producción de etanol a partir de almidón se lleva a cabo por la sacarificación de este polisacárido para convertirlo en azúcares fermentables, que son transformados en alcohol mediante levaduras [2, 3]; sin embargo, la concentración de alcohol producido oscila alrededor del $10 \% \mathrm{p} / \mathrm{p}$, lo cual hace necesario un costoso proceso adicional de purificación para alcanzar la concentración requerida $(>99 \% \mathrm{p} / \mathrm{p})$ para mezclarlo con gasolina en proporciones del 10 al 20\% p/p [4]; por tanto, el costo de producción de etanol anhidro es elevado y poco competitivo frente a los combustibles fósiles convencionales; pero la integración de procesos, por ejemplo, mediante la sacarificación-fermentación simultánea (SSF, por sus siglas en inglés), podría mejorar en forma significativa la productividad del proceso [5].

La SSF presenta la ventaja de simplificar el proceso, reduce el número de reactores necesarios, los tiempos de proceso, las demandas de energía y los costos de producción [6]; sin embargo, en este proceso se presenta inhibición del metabolismo de los microorganismos encargados de la fermentación; esto ocurre a una concentración de etanol del orden de $10 \% \mathrm{p} / \mathrm{p}$, lo que se traduce en una disminución de la actividad microbiana y, por ende, en el rendimiento del etanol [7]. Como solución a este problema se han planteado diferentes alternativas para la remoción in situ del etanol del medio fermentativo, de forma que la concentración de etanol se mantenga por debajo del valor inhibitorio; entre estas técnicas de remoción de etanol se destaca la pervaporación, proceso de separación de membranas que ha adquirido cada vez mayor interés, pues es eficiente desde el punto de vista energético, en comparación con tecnologías convencionales como la destilación y la adsorción [8].

En la pervaporación se pone una mezcla líquida en contacto con la superficie de una membrana, buscando que el compuesto que se va a separar se transporte preferentemente a través de ella; el lado de la membrana en contacto con la solución de trabajo se conoce como "retenido" o "alimento", y el otro lado, como "permeado"; sin embargo, pese a las múltiples ventajas económicas y técnicas que presenta este nuevo proceso de producción de alcohol, los fenómenos enzimáticos y de transporte de materia asociados son complejos; por esta razón, la modelación y simulación fundamentada en principios termodinámicos y cinéticos constituye una herramienta muy poderosa para determinar si el funcionamiento de este proceso es viable a nivel industrial; además, esta herramienta tiene otras ventajas, como encontrar las mejores condiciones de proceso, reduciendo así el trabajo experimental y mejorando los índices de desempeño ambiental. Por tanto, en este artículo se reporta la modelación y simulación de la sacarificación-fermentación simultánea de almidón acoplada a un pervaporador, empleando una aproximación fenomenológica; la solución de los modelos y su simulación se realiza con MatLab.

\section{Metodología}

\section{A. Modelo de la sacarificación-fermentación simultánea (SSF)}

La primera etapa del proceso es la sacarificación, donde se lleva a cabo la hidrólisis enzimática del almidón para la producción de la glucosa, que, posteriormente, pasa a la etapa de fermentación, donde se produce el etanol. Se han sugerido diferentes modelos para esta etapa, los cuales, generalmente, se basan en ecuaciones cinéticas del tipo MichaelisMenten, en el mecanismo multicadena y en la ecuación de Briggs-Haldane [9]. En este trabajo se utilizó el mecanismo multicadena, que se fundamenta en la ecuación de Michaelis-Menten o de equilibrio rápido estacionario, donde la transformación enzimática de sustrato $(S)$ a producto $(P)$ transcurre a través de la formación de un complejo enzimasustrato $(E S)$, en equilibrio con la enzima $(E)$ y el sustrato $(S)$, que se descompone dando origen a los productos de reacción $(P)$, Reacción 1. 
Modelación y simulación de un pervaporador acoplado a un proceso de sacarificación-fermentación para la producción de etanol

$$
E+S \underset{K_{2}}{\stackrel{K_{1}}{\longrightarrow}} E S \stackrel{K_{3}}{\rightarrow} E+P \quad \mathrm{R} 1
$$

En la etapa de sacarificación se utiliza igualmente un modelo basado en el mecanismo multicadena [10], donde se considera que el almidón se hidroliza completamente con $\alpha$-amilasa. Por tanto, el grado promedio de polimerización ( $D P$, por sus siglas en inglés) es 5, y es imposible alcanzar la completa conversión del almidón a glucosa [10]. Así mismo, se considera que los oligosacáridos están formados por dos fracciones: una susceptible a la hidrólisis, que comprende el $77 \%$ de las moléculas (enlaces $\alpha-1,4)$, y otra que contiene enlaces $\alpha-1,6$ que son resistentes a la hidrólisis [10]. El balance de masa para la fracción de almidón susceptible y resistente a la hidrólisis puede expresarse en forma diferencial, como se indica en las Ecuaciones (1) y (2) [11, 12] (en la Tabla 1 se muestra la definición de variables del modelo):

$$
\begin{aligned}
& \frac{d S_{s u s}}{d t}=-R_{s u s} S_{s u s} \\
& \frac{d S_{r e s}}{d t}=-R_{r e s} S_{r e s}
\end{aligned}
$$

Por tanto, la velocidad total de degradación del almidón es (3):

$$
\frac{d S}{d t}=-R_{s u s} S_{s u s}-R_{r e s} S_{r e s}
$$

Donde:

$$
R_{\text {sus }}=\frac{k_{\text {sus }} \text { Enz }}{K_{m}\left(1+\frac{G}{K_{G}}\right)+\frac{S_{\text {sus }}{ }^{2}}{K_{\text {starch }}}+S_{\text {sus }}+S_{\text {res }}}
$$

$$
R_{\text {res }}=\frac{k_{\text {res }} \text { Enz }}{K_{m}\left(1+\frac{G}{K_{G}}\right)+\frac{S_{r e s}^{2}}{K_{\text {starch }}}+S_{\text {sus }}+S_{\text {res }}}
$$

La concentración de las enzimas (Enz) depende de la velocidad de síntesis de enzimas $\left(R_{e n z}\right)$, de la velocidad de dilución del medio debido al crecimiento de la biomasa y de la velocidad de degradación de las enzimas $(\beta)$, Ecuación (6) $[13,14]$ :

$$
\frac{d E n z}{d t}=R_{e n z}-(\mu-\beta) E n z
$$

Donde:

$$
R_{\text {enz }}=\frac{\left(\mu_{m}+\beta\right)\left(S_{\text {sus }}+S_{\text {res }}\right) \text { Eenz }_{\max }}{K_{\text {Enz }}+S_{\text {sus }}+S_{\text {res }}}
$$

La velocidad de crecimiento de la biomasa se obtiene a partir del modelo de la etapa de la fermentación, en la cual se lleva a cabo la conversión de la glucosa y de los azúcares reductores, provenientes de la hidrólisis del almidón en etanol. Se han publicado diversos modelos de la fermentación de glucosa, los cuales generalmente se basan en ecuaciones tipo Monod, aproximación que también se usa en este trabajo. En concreto, el crecimiento del microorganismo que realiza la fermentación (Saccharomyces cerevisiae) puede expresarse como (8) [15]:

$$
\frac{d X}{d t}=\mu X-K_{d} X
$$

Donde,

$$
\mu=\mu_{m}\left(\frac{G}{G+k_{s}+\frac{G^{2}}{K_{S S}}}\right)\left(1-\frac{E}{E_{m}}\right)
$$


TABLA 1

DEFINICIÓN DE VARIABLES DEL MODELO DE SACARIFICACIÓN-FERMENTACIÓN SIMULTÁNEA

\begin{tabular}{|c|c|}
\hline VARIABLE & DEFINICIÓN DE VARIABLE \\
\hline & MODELO SACARIFICACIÓN FERMENTACIÓN SIMULTÁNEA \\
\hline $\mathrm{R}_{\text {sus }}$ & Velocidad de hidrolisis del almidón susceptible a ser hidrolizado $\left(\mathrm{h}^{-1}\right)$ \\
\hline $\mathrm{S}_{\text {sus }}$ & Concentración del almidón susceptible a ser hidrolizado (g/L) \\
\hline $\mathrm{R}_{\text {res }}$ & Velocidad de hidrolisis del almidón resistente ser hidrolizado $\left(\mathrm{h}^{-1}\right)$ \\
\hline $\mathrm{S}_{\mathrm{res}}$ & Concentración del almidón resistente a ser hidrolizado (g/L) \\
\hline $\mathrm{S}$ & Concentración de almidon (g/L) \\
\hline $\mathrm{k}_{\text {sus }}$ & Constante de velocidad de hidrólisis del almidón susceptible (g/Uh) \\
\hline $\mathrm{E}$ & Concentración del etanol (g/L) \\
\hline Enz & Concentración del enzima (g/L) \\
\hline $\mathrm{K}_{\mathrm{m}}$ & Constante de Michaelis (g/L) \\
\hline $\mathrm{G}$ & Concentración de la glucosa (g/L) \\
\hline $\mathrm{K}_{\mathrm{G}}$ & Constante de inhibición por la glucosa producida $(\mathrm{g} / \mathrm{L})$ \\
\hline $\mathrm{K}_{\text {starch }}$ & Constante de inhibición por el almidón \\
\hline $\mathrm{k}_{\mathrm{res}}$ & Constante de velocidad de hidrólisis del almidón resistente (g/Uh) \\
\hline $\mathrm{R}_{\mathrm{enz}}$ & Velocidad de síntesis de enzimas $\left(\mathrm{h}^{-1}\right)$ \\
\hline$\mu$ & Velocidad de producción de biomasa $\left(\mathrm{h}^{-1}\right)$ \\
\hline$\beta$ & Velocidad de degradación de las enzimas $\left(\mathrm{h}^{-1}\right)$ \\
\hline$\mu_{\mathrm{m}}$ & Velocidad máxima de crecimiento de la biomasas $\left(\mathrm{h}^{-1}\right)$ \\
\hline Eenz $_{\max }$ & Concentración máxima de enzima (U/L) \\
\hline $\mathrm{K}_{\mathrm{Enz}}$ & Constante de inhibición por la enzima (g/L) \\
\hline $\mathrm{X}$ & Concentración biomasa (g/L) \\
\hline $\mathrm{K}_{\mathrm{d}}$ & Constante de la velocidad de muerte de los microorganismos $\left(\mathrm{h}^{-1}\right)$ \\
\hline $\mathrm{k}_{\mathrm{s}}$ & Constante de saturación de la biomasa (g/L) \\
\hline $\mathrm{K}_{\mathrm{ss}}$ & Constante de inhibición por la biomasa (g/L) \\
\hline $\mathrm{E}_{\mathrm{m}}$ & Constante de inhibición por el etanol. (g/L) \\
\hline \multicolumn{2}{|r|}{ MODELO MEMBRANA PDMS } \\
\hline BAA & Coeficientes de fugacidad \\
\hline $\mathrm{D}_{\mathrm{Am}}$ & Difusividad del etanol $\left(\mathrm{m}^{2} / \mathrm{h}\right)$ \\
\hline $\mathrm{D}_{0}$ & Difusividaa a dilución infinita $\left(\mathrm{m}^{2} / \mathrm{h}\right)$ \\
\hline $\mathrm{E}^{*}$ & Energía necesaria para el salto. \\
\hline $\mathrm{j}_{\mathrm{A}}$ & Flux de etanol a través de la membrana $\left(\mathrm{kg} / \mathrm{m}^{2} \mathrm{~h}\right)$ \\
\hline $\mathrm{K}_{\mathrm{A}}^{\mathrm{G}}$ & Coeficiente del vapor $(1 / \mathrm{kpa})$ \\
\hline $\mathrm{K}_{\mathrm{A}}^{\mathrm{L}}$ & Coeficiente del líquido \\
\hline 1 & Espesor de la membrana (m) \\
\hline $\mathrm{M}_{\mathrm{A}}$ & Masa molar del etanol (kg/kmol) \\
\hline $\mathrm{P}_{\mathrm{A}}$ & Permeabilidad (kmol/m-h) \\
\hline $\mathrm{P}_{\mathrm{A}}^{\mathrm{G}}$ & Permeabilidad (kmol/m-h-Pa) \\
\hline
\end{tabular}




\begin{tabular}{|c|c|}
\hline VARIABLE & DEFINICIÓN DE VARIABLE \\
\hline $\mathrm{P}_{\text {Asat }}$ & Presión de saturación del etanol (kpa) \\
\hline $\mathrm{P}_{\mathrm{e}}$ & Presión del permeado (kpa) \\
\hline $\mathrm{P}_{\mathrm{o}}$ & Presión del alimento (kpa) \\
\hline $\mathrm{R}$ & Constante de los gases $\left(\mathrm{kpa}-\mathrm{m}^{3} / \mathrm{kmol}-\mathrm{K}\right)$ \\
\hline $\mathrm{t}$ & Tiempo de simulación (s) \\
\hline $\mathrm{T}$ & temperatura del sistema $(\mathrm{K})$ \\
\hline $\mathrm{S}_{\mathrm{Am}}$ & Solubilidad del etanol en la membrana \\
\hline $\mathrm{V}_{\mathrm{A}}$ & Volumen molar del etanol $\left(\mathrm{m}^{3} / \mathrm{kmol}\right)$ \\
\hline $\mathrm{V}_{1}^{*}$ & Volumen libre requerido para el salto del etanol \\
\hline $\mathrm{V}_{2}^{*}$ & Volumen libre especifico del polímero \\
\hline $\mathrm{V}_{\mathrm{FH}}^{*}$ & Volumen promedio libre especifico del PDMS puro. \\
\hline $\mathrm{X}_{\mathrm{Ae}}$ & Fracción molar del etanol en el permeado \\
\hline $\mathrm{X}_{\mathrm{Ae}(\mathrm{m})}$ & Fracción molar del etanol en la interfase inferior de la membrana \\
\hline $\mathrm{X}_{\text {Ao }}$ & Fracción molar del etanol en el alimento \\
\hline $\mathrm{X}_{\mathrm{Ao}}{ }^{\mathrm{G}}$ & Fracción molar del etanol en el vapor \\
\hline $\mathrm{X}_{\mathrm{Ao}}^{\mathrm{L}}$ & Fracción molar del etanol en el líquido \\
\hline $\mathrm{X}_{\mathrm{Ao}(\mathrm{m})}$ & Fracción molar del etanol en la interfase superior de la membrana \\
\hline$\gamma_{\mathrm{Ae}}$ & Coeficiente de actividad del etanol en el permeado \\
\hline$\gamma_{\mathrm{Ae}(\mathrm{m})}$ & Coeficiente de actividad del etanol en la interfase inferior de la membrana \\
\hline$\gamma_{\mathrm{Ao}}$ & Coeficiente de actividad del etanol en el alimento \\
\hline$\gamma_{\mathrm{Ao}}^{\mathrm{G}}$ & Coeficiente de fugacidad del etanol \\
\hline$\gamma_{\mathrm{Ao}}^{\mathrm{L}}$ & Coeficiente de actividad del etanol en el líquido \\
\hline$\gamma_{\mathrm{Ao}(\mathrm{m})}$ & Coeficiente de actividad del etanol en la interfase superior de la membrana \\
\hline$\alpha$ & Selectividad \\
\hline$\gamma$ & Factor de solapamiento \\
\hline$\delta_{\mathrm{AB}}$ & Coeficientes de fugacidad \\
\hline$\Lambda_{\mathrm{AB}}, \Lambda_{\mathrm{BA}}$ & Coeficientes de actividad \\
\hline$\mu_{\mathrm{Ao}}$ & Potencial químico del etanol en el alimento \\
\hline$\mu_{\mathrm{Ao}(\mathrm{m})}$ & Potencial químico del etanol en la interfase superior de la membrana \\
\hline$\mu_{\mathrm{A}}^{\mathrm{o}}$ & Potencial químico del etanol puro \\
\hline$\mu_{\mathrm{Ae}}$ & Potencial químico del etanol en el permeado \\
\hline$\mu_{\mathrm{Ae}(\mathrm{m})}$ & Potencial químico del etanol en la interfase inferior de la membrana \\
\hline$\mu_{\mathrm{Ao}}^{\mathrm{L}}$ & Potencial químico del etanol en el líquido \\
\hline$\mu_{\mathrm{Ao}}^{\mathrm{G}}$ & Potencial químico del etanol en el vapor \\
\hline$\xi$ & Relación entre el volumen critico del etanol y del polímero para el salto \\
\hline$\rho_{\mathrm{A}}$ & Concentración másica del etanol $\left(\mathrm{Kg} / \mathrm{m}^{3}\right)$ \\
\hline$\omega_{1}$ & Fracción masica del etanol \\
\hline$\omega_{2}$ & Fracción masica del polimero \\
\hline & MODELO MEMBRANA SILICALITA \\
\hline
\end{tabular}




\begin{tabular}{ll}
\hline VARIABLE & \multicolumn{1}{c}{ DEFINICIÓN DE VARIABLE } \\
\hline $\mathrm{A}^{\mathrm{s}}$ & Factor prexponecial difusividad Maxwell-Stefan $\left(\mathrm{m}^{2} / \mathrm{s}\right)$ \\
$\mathrm{D}^{\mathrm{S}}$ & Difusividad de Maxwell-Stefan $\left(\mathrm{m}^{2} / \mathrm{s}\right)$ \\
$\mathrm{E}^{\mathrm{s}}$ & Energía activación difusividad Maxwell-Stefan \\
$\mathrm{J}$ & Flux a través de la membrana $\left(\mathrm{Kg} / \mathrm{m}^{2} \mathrm{~s}\right)$ \\
$\mathrm{q}$ & Adsorción de la especie en la silicalita $(\mathrm{mol} / \mathrm{kg})$ \\
$\mathrm{q}_{\mathrm{M}}$ & Máxima posible adsorción de la especie en la silicalita $(\mathrm{mol} / \mathrm{kg})$ \\
$\mathrm{s}$ & Constante de Langmuir \\
$\mathrm{s}_{0}$ & Factor prexponecial constante de Langmuir \\
$\mathrm{v}$ & Velocidad de difusión de las especies $(\mathrm{m} / \mathrm{s})$ \\
$\mu$ & Potencial químico \\
$\varepsilon$ & Porosidad de la silicalita (um) \\
$\rho_{\mathrm{s}}$ & Densidad de la silicalita $\left(\mathrm{Kg} / \mathrm{m}^{3}\right)$ \\
$\theta_{\mathrm{K}}$ & Fracción de sitios ocupados ocupados por la especie $\mathrm{k}$ \\
$\theta_{\mathrm{n}+1}$ & Representa la fracción de sitios vacantes en la zeolita \\
$\theta_{\mathrm{V}}$ & Fracción de sitios vacantes \\
\hline
\end{tabular}

\section{B. Modelo del proceso de pervaporación}

\section{1) Modelado de las membranas de PDMS}

En primera instancia se modeló el transporte de etanol a través de membranas PDMS en estado estacionario. El modelo fenomenológico empleado fue el modelo de solución-difusión. Luego se incluyó la variación temporal de la concentración de etanol que se presenta durante la fermentación; este cambio en la concentración de etanol está determinado por la velocidad de producción de etanol en el proceso de la sacarificación-fermentación simultánea (SSF) y la velocidad de permeación del etanol a través de la membrana. Las consideraciones del modelo son las siguientes:

- Mezcla binaria etanol-agua.

- La etapa que gobierna el transporte de masa es la difusión a través de la membrana.

- Sistema en estado no estacionario.

- No hay transporte de masa por convección.

- La concentración de alimentación y todas las propiedades que dependen de esta (como el coeficiente de actividad, la densidad de la mezcla, la permeabilidad, la solubilidad de la mezcla en la membrana y el coeficiente de distribución) son dependientes del tiempo.

El desarrollo del modelo en estado no estacionario para la membrana PDMS se resume a continuación (referirse en la Tabla 1 para la definición de variables del modelo). Partiendo de la ecuación de Fick se tiene que el flux másico del etanol $\left(\mathrm{j}_{A}(t)\right)$ a través de la membrana es directamente proporcional al coeficiente de difusión en la membrana e inversamente proporcional a su espesor (10):

$$
\mathrm{j}_{\mathrm{A}}(t)=-\mathrm{D}_{\mathrm{A}} \frac{\left.\mathrm{d} \rho_{\mathrm{A}}(t)\right)}{\mathrm{dl}}
$$

Considerando que, por definición, $\left.\rho_{\mathrm{A}}(t)\right)=M_{A} C_{A}(t) \mathrm{y}$ $C_{A}(t)=C \mathrm{x}_{\mathrm{A}}(t)$, se tiene (11):

$$
\mathrm{j}_{\mathrm{A}}(t)=\frac{\mathrm{M}_{\mathrm{A}} \mathrm{CD}_{\mathrm{A}}}{\ell}\left(\mathrm{x}_{\mathrm{Ao}(\mathrm{m})}(t)-\mathrm{x}_{\mathrm{Ae}(\mathrm{m})}\right)
$$

La fracción molar de etanol en la superficie superior de la membrana $\left(x_{A 0(m)}\right)$ puede relacionarse con su concentración en el fermentador $\left(x_{A 0}\right)$ mediante un balance de masa en la interfase superior de la membrana. De igual forma, la fracción molar de etanol en la superficie inferior $\left(x_{A e(m)}\right)$ puede relacionarse con su concentración en el permeado 
Modelación y simulación de un pervaporador acoplado a un proceso de sacarificación-fermentación para la producción de etanol

$\left(x_{A e}\right)$ mediante un balance de masa en la interfase inferior de la membrana. Además, puesto que en el proceso se llega a un equilibrio líquido-vapor, deben involucrarse los coeficientes de actividad $\left(\gamma_{A}\right)$. El resultado final se muestra en (12); los detalles de la derivación se presentan como material complementario al artículo.

$$
\mathrm{j}_{\mathrm{A}}(t)=\frac{\mathrm{M}_{\mathrm{A}} \mathrm{P}_{\mathrm{A}}(t)}{\ell}\left(\mathrm{x}_{\mathrm{Ao}}(t)-\frac{\gamma_{\mathrm{Ao}}^{\mathrm{G}}(t)}{\gamma_{\mathrm{Ao}}{ }^{\mathrm{L}}(t)} \frac{\mathrm{P}_{\mathrm{e}}}{\mathrm{P}_{\mathrm{Asat}}} \mathrm{x}_{\mathrm{Ae}} \exp \left(\frac{-\mathrm{v}_{\mathrm{A}}\left(\mathrm{P}_{\mathrm{o}}-\mathrm{P}_{\mathrm{Asat}}\right)}{\mathrm{RT}}\right)\right)
$$

Donde $P_{A}$ representa la permeabilidad del etanol en la membrana, la cual depende de su solubilidad $\left(S_{A m}\right)$ y coeficiente de difusión $\left(D_{A m}\right)$ en la membrana (13):

$$
\mathrm{P}_{\mathrm{A}}(t)=\mathrm{D}_{\mathrm{Am}} S_{A m}(t)
$$

La difusividad del etanol en la membrana puede ser expresada por la teoría del transporte de volumen libre [16] como (14):

$$
\mathrm{D}_{A m}=\mathrm{D}_{0} \exp \left[\frac{-\mathrm{E} *}{\mathrm{RT}}\right] \exp \left[\frac{-\omega_{1} V_{1}^{*}+\omega_{2} \xi V_{2} *}{V_{F H} / \gamma}\right]
$$

Utilizando la ecuación del virial truncada hasta el segundo término y el modelo de UNIQUAC para evaluar los coeficientes de fugacidad y de actividad, (15) y (16), respectivamente, del etanol se tiene:

$$
\begin{gathered}
\gamma_{\mathrm{Ao}}{ }^{\mathrm{G}}(t)=\exp \left[\frac{\mathrm{P}_{\mathrm{o}}}{\mathrm{RT}}\left(\mathrm{B}_{\mathrm{AA}}+\mathrm{x}_{\mathrm{Ao}}{ }^{\mathrm{G}}(t) \delta_{\mathrm{AB}}\right)\right] \\
\gamma_{\mathrm{Ao}} \mathrm{L}(t)=\exp \left[\begin{array}{l}
-\ln \left(\mathrm{x}_{\mathrm{Ao}}(t)+\left(1-\mathrm{x}_{\mathrm{Ao}}(t)\right) \Lambda_{\mathrm{AB}}\right)+ \\
\left.\left(1-\mathrm{x}_{\mathrm{Ao}}(t)\right)\left(\frac{\Lambda_{\mathrm{AB}}}{\mathrm{x}_{\mathrm{Ao}}(t)+\left(1-\mathrm{x}_{\mathrm{Ao}}(t)\right) \Lambda_{\mathrm{AB}}}-\frac{\Lambda_{\mathrm{BA}}}{\left(1-\mathrm{x}_{\mathrm{Ao}}(t)\right)+\mathrm{x}_{\mathrm{Ao}}(t) \Lambda_{\mathrm{BA}}}\right)\right]
\end{array}\right.
\end{gathered}
$$

Introduciendo la definición de selectividad $(\alpha)$, Ecuación 17, el flux de etanol a través de la membrana está dado por (18).

$$
\mathrm{j}_{\mathrm{A}}(t)=\frac{\mathrm{M}_{\mathrm{A}} \mathrm{D}_{\mathrm{Am}} S_{A m}(t)}{\ell} \times
$$

$$
\mathrm{x}_{\mathrm{Ae}}(t)=\frac{\alpha}{1 / \mathrm{x}_{\mathrm{Ao}}(t)^{+\alpha-1}}
$$

$$
\left(\begin{array}{l}
\mathrm{x}_{\mathrm{Ao}}(t)-\frac{\exp \left[\frac{\left.\mathrm{P}_{\mathrm{o}}\left(\mathrm{B}_{\mathrm{AA}}+\mathrm{x}_{\mathrm{Ao}}{ }^{\mathrm{G}}(t) \delta_{\mathrm{AB}}\right)\right]}{\mathrm{RT}}\left[-\ln \left(\mathrm{x}_{\mathrm{Ao}}(t)+\left(1-\mathrm{x}_{\mathrm{Ao}}(t)\right) \Lambda_{\mathrm{AB}}\right)+\left(1-\mathrm{x}_{\mathrm{Ao}}(t)\right)\left(\frac{\Lambda_{\mathrm{AB}}}{\mathrm{x}_{\mathrm{Ao}}(t)+\left(1-\mathrm{x}_{\mathrm{Ao}}(t)\right) \Lambda_{\mathrm{AB}}}-\frac{\Lambda_{\mathrm{BA}}}{\left(1-\mathrm{x}_{\mathrm{Ao}}(t)\right)+\mathrm{x}_{\mathrm{Ao}}(t) \Lambda_{\mathrm{BA}}}\right)\right]\right.}{\left(\frac{\mathrm{P}_{\mathrm{e}}}{\mathrm{P}_{\mathrm{Asat}}}\right)\left(\frac{\alpha}{1 / \mathrm{x}_{\mathrm{Ao}}(t)+\alpha-1}\right) \exp \left(\frac{-\mathrm{v}_{\mathrm{A}}\left(\mathrm{P}_{\mathrm{o}}-\mathrm{P}_{\mathrm{Asat}}\right)}{\mathrm{RT}}\right)}
\end{array}\right)
$$




\section{2) Modelado de las membranas de silicalita}

Por tratarse de un material microporoso, se empleó el modelo de adsorción-difusión, que considera la adsorción de los componentes de una mezcla en la superficie de la membrana, y su posterior difusión a través de esta gracias a un gradiente de potencial químico, seguida por la desorción en forma de vapor de los compuestos que permearon (Fig. 1). La difusión dentro de la membrana se basa en las ecuaciones de Maxwell-Stefan [17].
Las simplificaciones implementadas en el desarrollo del modelo son las siguientes:

- Mezcla binaria etanol-agua.

- La etapa que gobierna el transporte de masa es la difusión del etanol en los microporos de la zeolita.

- $\quad$ El etanol es adsorbido en mayor cantidad por la zeolita con respecto al agua.

- $\quad$ Sistema en estado No Estacionario.

- La temperatura a través de la membrana es constante.

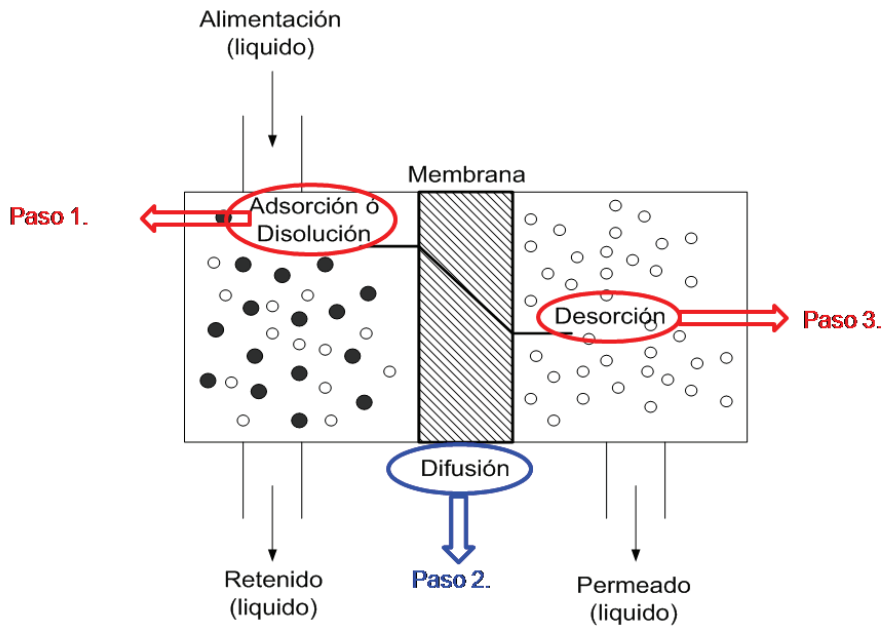

Fig. 1. Diagrama modelo adsorción-difusión para la membrana de silicalita.

En las ecuaciones de difusión multicomponente de Maxwell-Stefan se tienen en cuenta las interacciones entre la difusión de los diferentes componentes de la mezcla, como se muestra en (19):

$$
\nabla \mu_{i}(t)=R T \sum_{k=1, k \neq i}^{n} \theta_{K}(t) \frac{\left(v_{i}(t)-v_{k}(t)\right)}{D_{i k}^{s}(t)}+R T \theta_{n+1}(t) \frac{\left(v_{i}(t)-v_{n+1}(t)\right)}{D_{i, n+1}^{s}(t)} \text { para } i=1,2, \ldots n
$$

Es importante destacar que debido a que la concentración de etanol en la superficie de la membrana varía con el tiempo, la fracción de sitios ocupados y la difusividad superficial también son dependientes del tiempo. La fracción de sitios ocupados se define como:

$$
\theta_{i}(t)=\frac{q_{i}(t)}{q_{M, i}}
$$

Ahora, el flux para la especie $i$ a través de la membrana de la zeolita se puede definir en función de su velocidad:

$$
J_{i}(t)=\rho_{z} \varepsilon q_{M, i} \theta_{i}(t) \nu_{i}(t)
$$

Reemplazando (21) en (19) y reordenando términos para el sistema binario etanol-agua y teniendo en cuenta que para membranas de zeolita que muestran una alta selectividad, como es el caso de la silicalita, se considera que principalmente un componente permea a través de la membrana, se tiene: 
Modelación y simulación de un pervaporador acoplado a un proceso de sacarificación-fermentación para la producción de etanol

$$
-\frac{\nabla \mu_{1}(t)}{R T}=\left[\frac{\theta_{2}(t)\left(\frac{J_{1(t)}}{q_{M, 1} \theta_{1}(t)}-\frac{J_{2}(t)}{q_{M, 2} \theta_{2}(t)}\right)}{\rho_{z} \varepsilon D^{s}{ }_{12}(t)}+\frac{J_{1}(t)}{\rho_{z} \varepsilon q_{M, 1} \theta_{1}(t) D^{s}{ }_{1 V}(t)}\right]
$$

Donde la especie 1 es el etanol.

Suponiendo que la difusión acoplada de las especies $\left(D_{12}^{s}\right)$ tiende a infinito,(29) se convierte en:

$$
-\frac{\nabla \mu_{1}(t)}{R T}=\frac{J_{1}(t)}{\rho_{z} \varepsilon q_{M, 1} \theta_{1}(t) D_{1 V}^{s}(t)}
$$

El gradiente de potencial químico $\left(\nabla \mu_{l}\right)$ puede ser representado como el gradiente de la ocupación del sitio de cada especie [18]:

$$
-\frac{\nabla \mu_{1}(t)}{R T}=\theta_{1}(t) \frac{\partial \ln a_{1}(t)}{\partial \theta_{1}(t)} \frac{\partial \theta_{1}(t)}{\partial l}+\theta_{1}(t) \frac{\partial \ln a_{1}(t)}{\partial \theta_{2}(t)} \frac{\partial \theta_{2}(t)}{\partial l}
$$

Donde la actividad (a) depende de la fracción de sitios ocupados y, por tanto, es función del tiempo.Igualando (23) y (24) se obtiene:

$$
J_{1}(t)=-\rho_{z} \varepsilon q_{M, 1} \theta_{1}(t) D_{1 V}^{s}(t)\left[\theta_{1}(t) \frac{\partial \ln a_{1}(t)}{\partial \theta_{1}(t)} \frac{\partial \theta_{1}(t)}{\partial l}+\theta_{1}(t) \frac{\partial \ln a_{1}(t)}{\partial \theta_{2}(t)} \frac{\partial \theta_{2}(t)}{\partial l}\right]
$$

La ecuación 25 describe el flux en función del tiempo de cada uno de los componentes a través de los poros de la zeolita. La naturaleza de las funciones $\left(\partial \ln a_{1}(\mathrm{t}) / \partial \theta_{1}(\mathrm{t})\right)$ y $\left(\partial \ln a_{1}(\mathrm{t}) / \partial \theta_{2}(\mathrm{t})\right)$ dependerá de la naturaleza de la isoterma de adsorción del etanol en la zeolita. La difusividad $D_{l v}^{s}(\mathrm{t})$ también es dependiente de la fracción de los sitios ocupados. Por tanto, para modelar el flujo de cada uno de los componentes a través de la zeolita deben conocerse las características de difusión y adsorción. En este sentido, debido al estrecho diámetro de poro de la silicalita puede suponerse que solamente una molécula se difunde a la vez. Por tanto, la difusividad de Maxwell-Stefan $D_{l v}^{s}(t)$ depende linealmente de los sitios vacantes $\left(\theta_{\mathrm{v}}\right),(26)$ y $(27)[19]$ :

$$
\begin{gathered}
D^{S}{ }_{1 V}(t)=\frac{D^{S} 1, n+1}{\theta_{n+1}(t)} \\
D^{s}{ }_{i V}(t)=D^{s}{ }_{i V}(0) \theta_{V}(t)
\end{gathered}
$$

Por otro lado, la difusividad a dilución infinita depende de la temperatura, de acuerdo con la ecuación de Arrhenius (28) [20]:

$$
D^{s}{ }_{\nu V}(0)=A_{i}^{s} \exp \left(-\frac{E_{i}{ }^{s}}{R T}\right)
$$

Asumiendo que la adsorción del etanol se pude describir con la isoterma de adsorción de Langmuir, la actividad $\left(a_{l}(t)\right)$ en la zeolita para una determinada fracción de ocupación del sitio $\left(\theta_{I}(t)\right)$ está dada por (29):

$$
a_{1}(t)=\frac{s \theta_{1}(t)}{1-\theta_{1}(t)}
$$

Donde la constante de Langmuir $(s)$ es función de la temperatura. 
Finalmente, el flux de etanol a través de la membrana está dado por (30):

$$
J_{1}=-\rho_{z} \varepsilon q_{M, 1} \theta_{1} D^{S} 1 V(0) \frac{\partial \theta_{1}}{\partial l}
$$

\subsubsection{Modelo de la membrana compuesta PDMS + silicalita}

También se modelaron membranas compuestas PDMS y Silicalita. Un primer caso es cuando una capa de PDMS se deposita sobre una capa de silicalita (la película de PDMS está en la parte superior debido a su mayor resistencia al envenenamiento por los subproductos de la fermentación); en este caso puede emplearse un modelo de resistencias en serie, como se ilustra en la Fig. 2. En este modelo, el fluxa través de la capa de PDMS es el mismo que a través de la silicalita, pudiendo igualarse (18) y (30) para calcular la concentración del etanol en la interface de las capas y a la salida de la membrana compuesta (concentración de permeado).

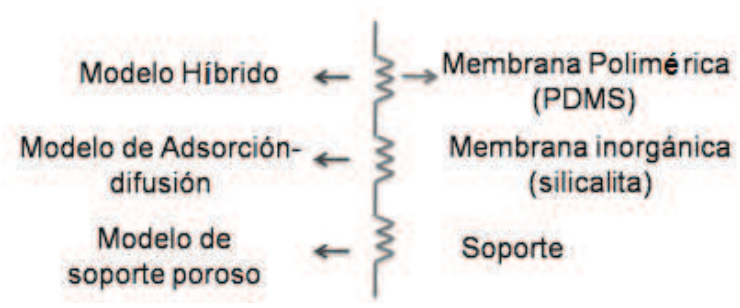

Fig. 2. Esquema del modelo de resistencia en serie para la membrana compuesta por capas PDMS-Silicalita.

\section{RESUlTADOS Y DISCUSIÓN}

\section{A. Simulación del modelo sacarificación- fermentación simultánea}

El modelo de la SSF se implementó en MatLab. Los resultados del modelo se compararon con los obtenidos por el Grupo de Biotransformación, de la Universidad de Antioquia, para la producción de etanol a partir de almidón de yuca; las condiciones bajo las cuales se realizaron los experimentos son: biorreactor de $5 \mathrm{~L}$, enzima AMG y Termamil, levadura ETHANOL RED, temperatura: $36^{\circ} \mathrm{C}, \mathrm{pH}: 4.5$.

El ajuste del modelo a los resultados experimentales es muy bueno (Fig. 4 a 6); en efecto, la desviación máxima encontrada entre los valores experimentales y simulados es del 3\%. Puesto que lo más importante del modelo acoplado es determinar la concentración de etanol en función del tiempo, a continuación se muestra el efecto de diferentes parámetros de operación sobre el desempeño del sistema:

- $\quad$ Concentración de enzima: Al aumentar la concentración de la enzima, la producción de etanol permanece prácticamente constante (Fig. 3); esto se debe a que la mayor concentración de enzima aumenta la velocidad de hidrólisis del almidón, lo que incrementa la concentración de glucosa y azúcares reductores. Este resultado coincide con el hallado en la simulación de la etapa de fermentación (no mostrado), donde el aumento en la concentración de la glucosa no influye en la producción del etanol.
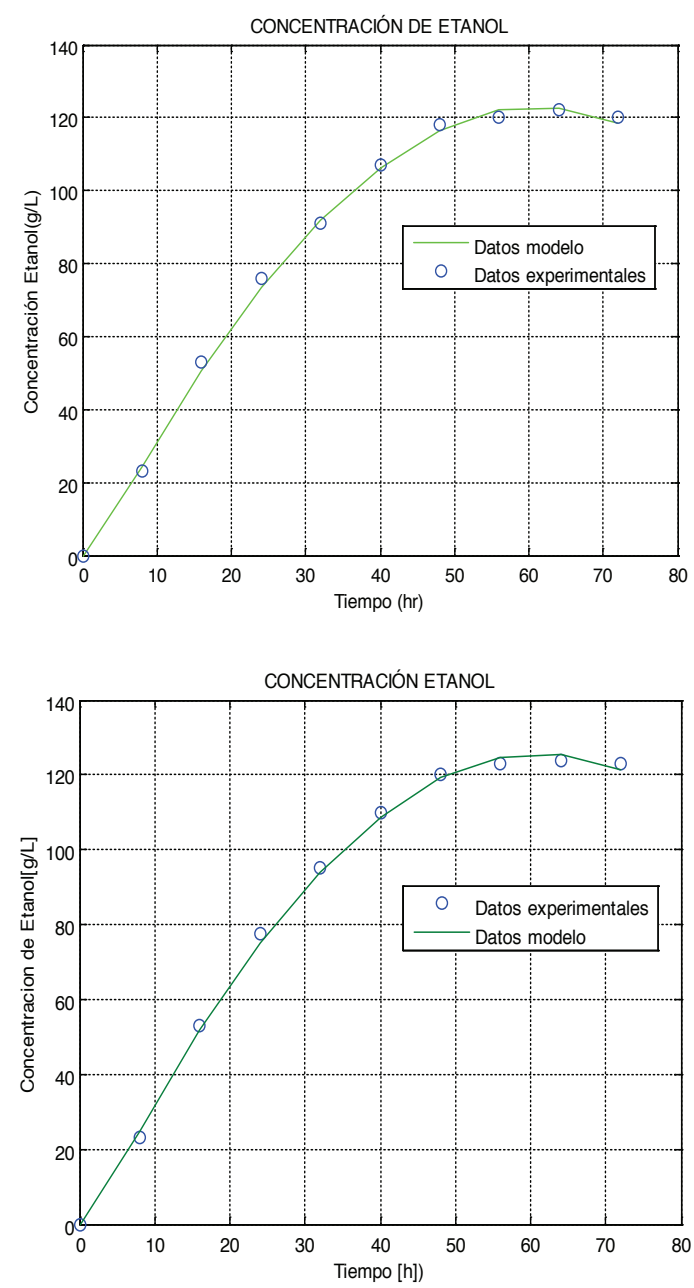

Fig. 3. Efecto de la variación de la concentración de enzima ( $1 \mathrm{~mL} / \mathrm{L}$-izquierda y $2 \mathrm{~mL} / \mathrm{L}$-derecha) a $\mathrm{pH}$ (4) y concentración de inóculo $(0.25 \mathrm{~g} / \mathrm{L})$ constantes. 
Modelación y simulación de un pervaporador acoplado a un proceso de sacarificación-fermentación para la producción de etanol

- $\quad$ Concentración del inóculo: Al aumentar la concentración del inóculo, aumenta en el tiempo la concentración del etanol (Fig. 4), debido a que hay mayor número de microorganismos que realizan la fermentación, y, por tanto, la glucosa y los azúcares reductores se convierten más rápidamente en etanol.
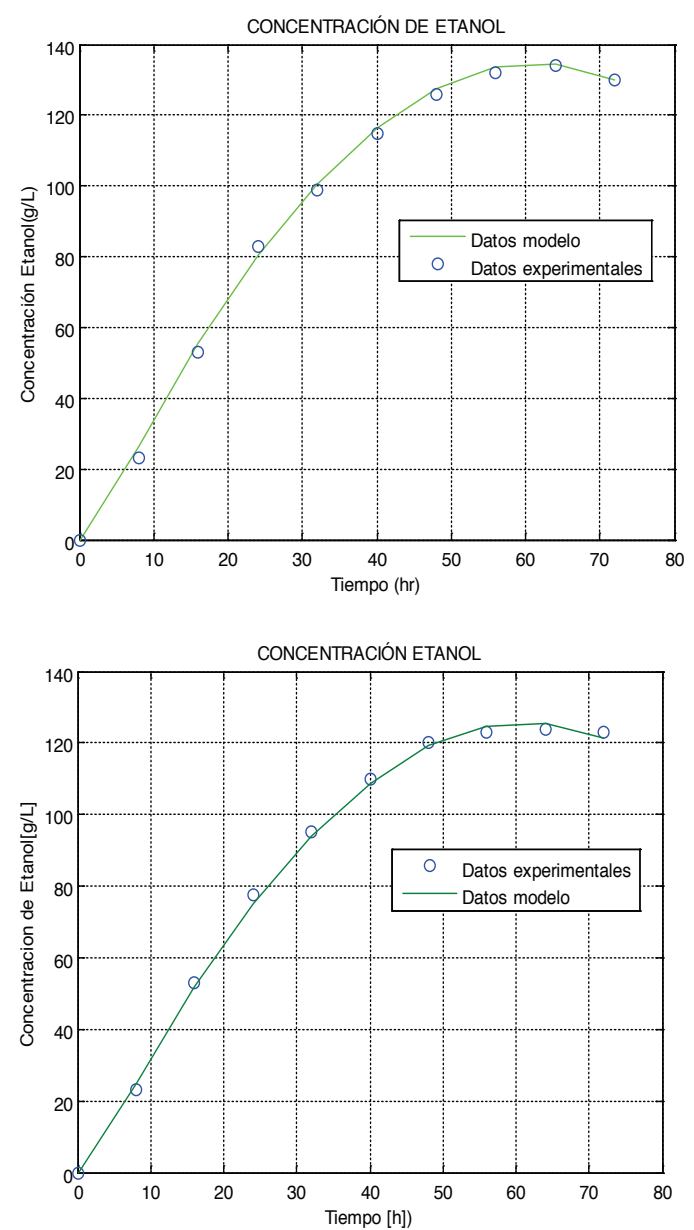

Fig. 4. Efecto de la variación de la concentración de inóculo $(0.25 \mathrm{~g} / \mathrm{L}$-izquierda y $0.75 \mathrm{~g} / \mathrm{L}$-derecha) a $\mathrm{pH}$ (4) y concentración de enzima ( $2 \mathrm{~mL} / \mathrm{L})$ constantes.

- $\quad$ pH: Al aumentar el pH, la concentración del etanol disminuye (Fig. 5), puesto que $\mathrm{pH}$ altos favorecen la producción de azúcares reductores, disminuyendo la eficiencia de los microorganismos encargados de la fermentación [5].
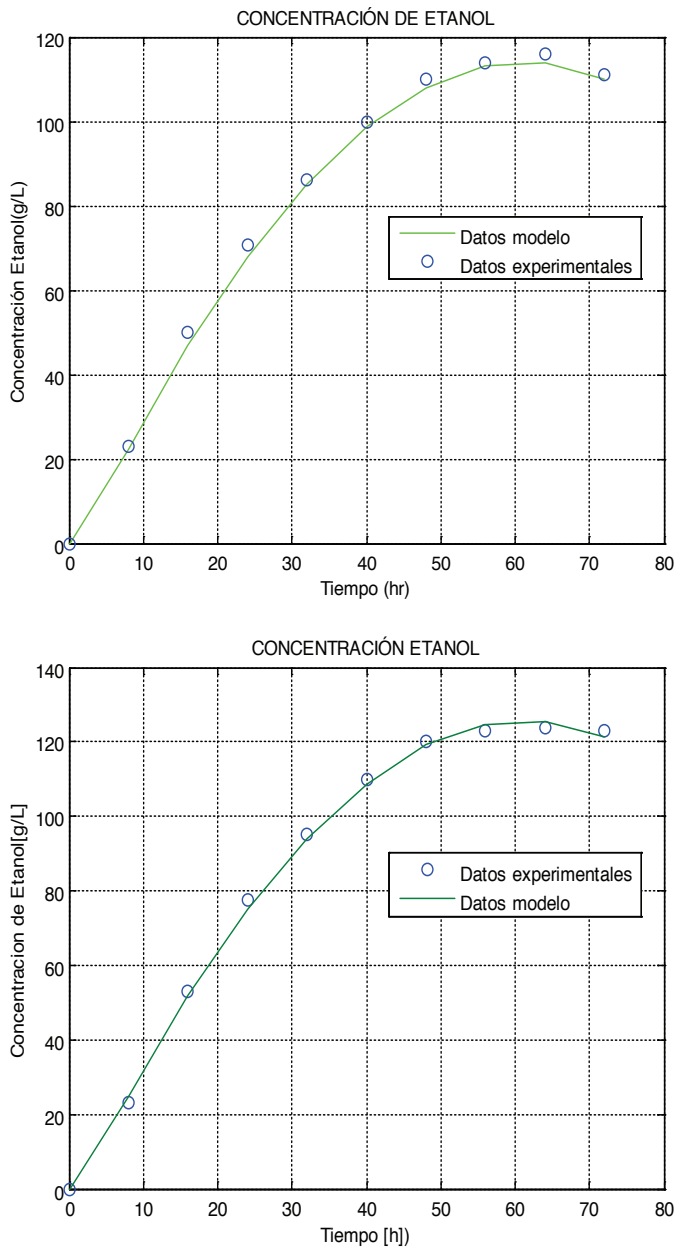

Fig. 5. Efecto de la variación del pH (4-izquierday 5-derecha-) a concentración de enzima $(1 \mathrm{~mL} / \mathrm{L})$ y concentración de inóculo $(0.25 \mathrm{~g} / \mathrm{L})$ constantes.

\section{B. Simulación del modelo de pervaporación}

1) Simulación del modelo de las membranas de PDMS y silicalita

Los modelos de solución-difusión y adsorción difusión arrojaron un buen ajuste a los datos experimentales reportados para la separación de etanol con membranas de PDMS y silicalita [2027], respectivamente (ver Fig. 6). Las condiciones experimentales empleadas en cada referencia se indican en la Tabla 2; estas condiciones se usaron como parámetros de entrada en la simulación. 

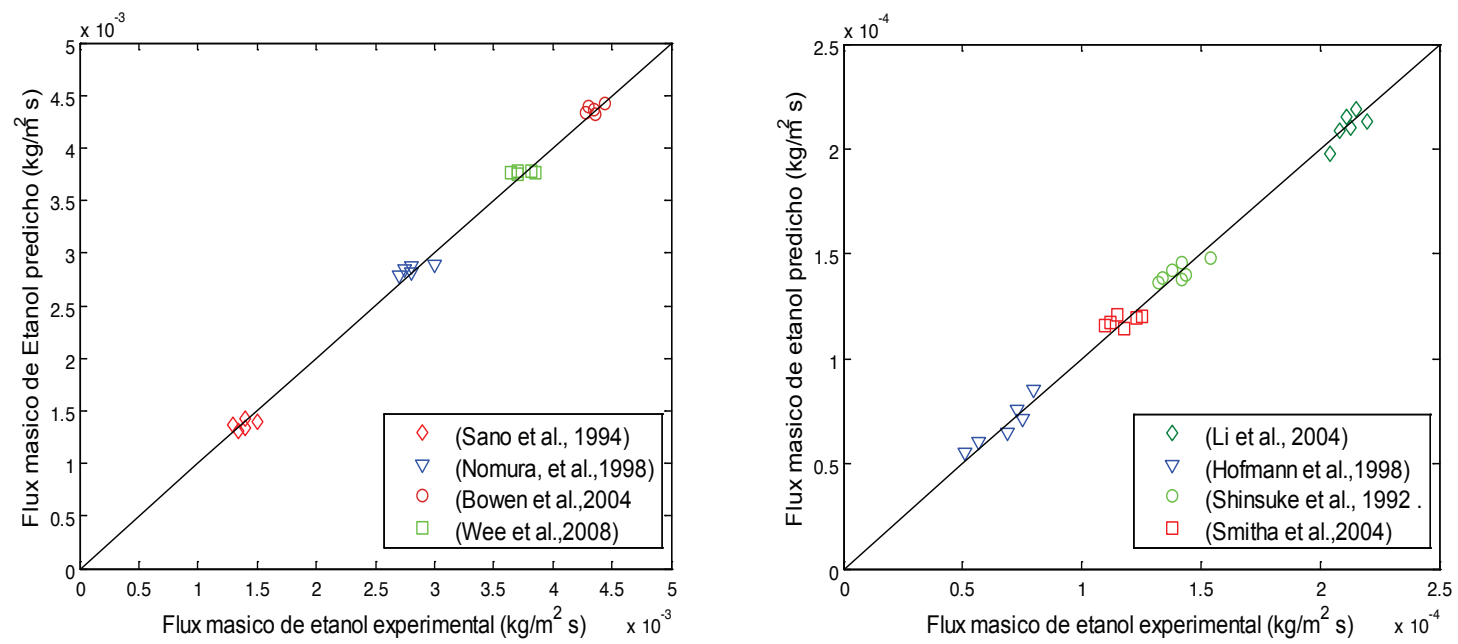

Fig.6. Validación del modelo de transporte de etanol en membranas de PDMS (izq.) y silicalita (der.)

TABLA 2

CONDICIONES EXPERIMENTALES DE LOS ESTUDIOS USADOS PARA LA VALIDACIÓN DE LOS MODELOS DE LAS MEMBRANAS DE PDMS Y SILICALITA

\begin{tabular}{|l|c|c|c|c|}
\hline \multicolumn{5}{|c|}{ MEMBRANA PDMS } \\
\hline \multicolumn{1}{|c|}{ Referencia } & $\begin{array}{c}\text { Shinsuke } \\
(1992)\end{array}$ & $\begin{array}{c}\text { Hofmann } \\
(1998)\end{array}$ & $\begin{array}{c}\text { Li } \\
(2004)\end{array}$ & $\begin{array}{c}\text { Smitha } \\
(2004)\end{array}$ \\
\hline $\begin{array}{l}\text { Temp. alimento } \\
\left({ }^{\circ} \mathrm{C}\right)\end{array}$ & 35 & 35 & 60 & 35 \\
\hline $\begin{array}{l}\text { Presión vacío } \\
(\mathrm{mmHg})\end{array}$ & 5 & 15 & 10 & 10 \\
\hline $\begin{array}{l}\text { Espesor } \\
\text { membrana( } \mu \mathrm{m})\end{array}$ & 10 & 25 & 15 & 10 \\
\hline $\begin{array}{l}\text { Área membrana } \\
\left.(\mathrm{cm})^{2}\right)\end{array}$ & 20 & 10 & 25 & 20 \\
\hline $\begin{array}{l}\text { Caudal } \\
\text { alimentación (ml/ } \\
\text { min) }\end{array}$ & 100 & 50 & 150 & 100 \\
\hline \multicolumn{1}{|c|}{ Referencia } & Sano (1994) & $\begin{array}{c}\text { Nomura } \\
(1998)\end{array}$ & $\begin{array}{c}\text { Bowen } \\
(2004)\end{array}$ & $\begin{array}{c}\text { Wee } \\
(2004)\end{array}$ \\
\hline $\begin{array}{l}\text { Temp. alimento } \\
\left({ }^{\circ} \mathrm{C}\right)\end{array}$ & 25 & 30 & 55 & 35 \\
\hline $\begin{array}{l}\text { Presión vacio } \\
(\text { mmHg) }\end{array}$ & 10 & 15 & 5 & 10 \\
\hline $\begin{array}{l}\text { Espesor } \\
\text { membrana( } \mu \mathrm{m})\end{array}$ & 100 & 70 & 50 & 20 \\
\hline $\begin{array}{l}\text { Área Membrana } \\
\left(\mathrm{cm}{ }^{2}\right)\end{array}$ & 20 & 10 & 25 & 20 \\
\hline $\begin{array}{l}\text { Caudal } \\
\text { alimentación (ml/ } \\
\text { min) }\end{array}$ & 80 & 120 & 200 & 100 \\
\hline
\end{tabular}


Modelación y simulación de un pervaporador acoplado a un proceso de sacarificación-fermentación para la producción de etanol

2) Simulación del modelo de las membranas compuestas de PDMS-Silicalita

Inicialmente, el modelo por capas se validó con los resultados reportados por diferentes autores. Los resultados se muestran en la Fig. 7, donde las líneas continuas son las predicciones del modelo, y los puntos son los valores experimentales reportados [28-30]; las condiciones experimentales empleadas en cada referencia se indican en la Tabla 3. Es importante destacar que en el modelo se tomaron los datos experimentales de concentración de etanol en función del tiempo reportados por cada autor; es decir, no se utilizó el modelo de sacarificaciónfermentación simultáneas. En todos los casos se encontró una diferencia menor al $2 \%$ entre los datos calculados con el modelo y los reportados en la literatura.

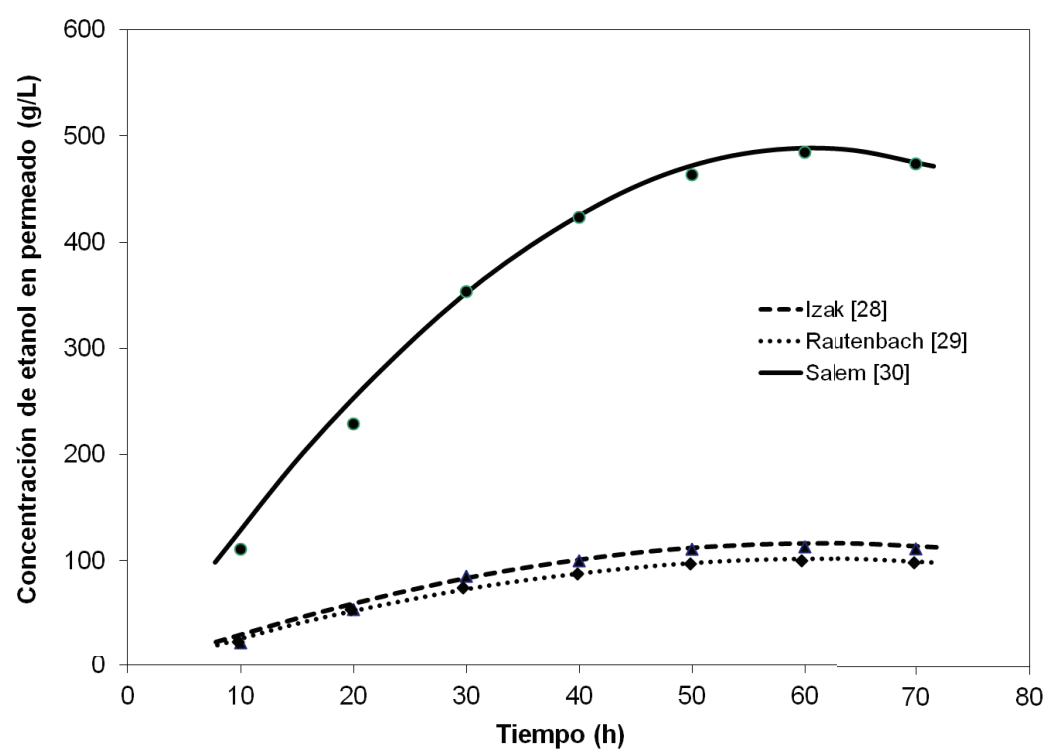

Fig. 7. Validación del modelo de transporte de etanol en membranas compuestas por capas PDMS-Silicalita.

TABLA 3

CONDICIONES EXPERIMENTALES DE LOS ESTUDIOS USADOS PARA LA VALIDACIÓN DEL MODELO DE LA MEMBRANA COMPUESTA POR CAPAS PDMS-SILICALITA

\begin{tabular}{|c|c|c|c|}
\hline Referencia & $\begin{array}{c}\text { Izak } \\
(2003)\end{array}$ & $\begin{array}{c}\text { Rautenbach } \\
(2003)\end{array}$ & $\begin{array}{c}\text { Salem } \\
(2006)\end{array}$ \\
\hline $\begin{array}{c}\text { Temperatura } \\
\text { alimento }\left({ }^{\circ} \mathrm{C}\right)\end{array}$ & 35 & 35 & 60 \\
\hline $\begin{array}{c}\text { Presión vacío } \\
(\mathrm{mmHg})\end{array}$ & 15 & 20 & 5 \\
\hline $\begin{array}{c}\text { Espesor capa } \\
\text { PDMS }(\mu \mathrm{m})\end{array}$ & 25 & 50 & 10 \\
\hline $\begin{array}{c}\text { Espesor capa } \\
\text { Silicalita }(\mu \mathrm{m})\end{array}$ & 100 & 50 & 50 \\
\hline $\begin{array}{c}\text { Área membrana } \\
\left(\mathrm{cm}^{2}\right)\end{array}$ & 20 & 25 & 30 \\
\hline $\begin{array}{c}\text { Caudal alimentación } \\
\left(\mathrm{ml} / \mathrm{min}^{2}\right)\end{array}$ & 100 & 50 & 150 \\
\hline
\end{tabular}


Con el modelo validado se estudió el efecto de diferentes condiciones de operación (temperatura del alimento, presión de vacío, espesor de la membrana de PDMS y silicalita) sobre el desempeño de la separación de etanol en mezclas modelo etanolagua. A continuación se reseñan las principales observaciones encontradas:

- $\quad$ Temperatura. Un aumento de la temperatura del alimento, dejando los demás parámetros constantes, causa un aumento significativo en la concentración del etanol en el permeado; esto se debe a dos factores: a) el coeficiente de difusividad en la capa de PDMS es directamente proporcional a la temperatura (ver Ecuación 14), y, por consiguiente, en esta capa se puede separar más etanol de la mezcla de alimentación, y b) el coeficiente de adsorción en la capa de silicalita también es directamente proporcional a la temperatura (ver Ecuación 28); la mayor adsorción se traduce en un mayor flux de etanol en esta capa.

- $\quad$ Presión de vacío aplicada en el lado del permeado. Aunque al aumentar la presión de vacío del sistema, dejando los demás parámetros constantes, la concentración de etanol en el permeado aumenta de forma significativa, es importante destacar que en las membranas de PDMS y silicalita la contribución de la presión de vacío en el aumento del flux de etanol es la misma.

- $\quad$ Espesor de cada capa. La disminución del espesor de la película de PDMS es técnicamente difícil, pues una membrana de $5 \mu \mathrm{m}$ (espesor considerado en la simulación) se considera ultrafina. Sin embargo, si este espesor se redujera a 2 $\mu \mathrm{m}$, se lograría un aumento significativo (superior al $50 \%$ ) en el flux a través de la membrana (en ambos casos se consideró que el espesor de la membrana de silicalita es de $100 \mu \mathrm{m})$. El efecto de la disminución del espesor de la membrana de silicalita no es tan significativo como en el caso de la capa de PDMS. De hecho, una reducción de un orden de magnitud en el espesor de la membrana de silicalita (pasando de 100 a $10 \mu \mathrm{m}$ ), causa un aumento del flux de etanol a través de la membrana, del mismo orden que el observado al reducir el espesor de la membrana de PDMS de 5 a $2 \mu \mathrm{m}$ (es decir, del orden del 50\%).

\section{Simulación del modelo del proceso} sacarificación-fermentación simultánea acopladoal pervaporador

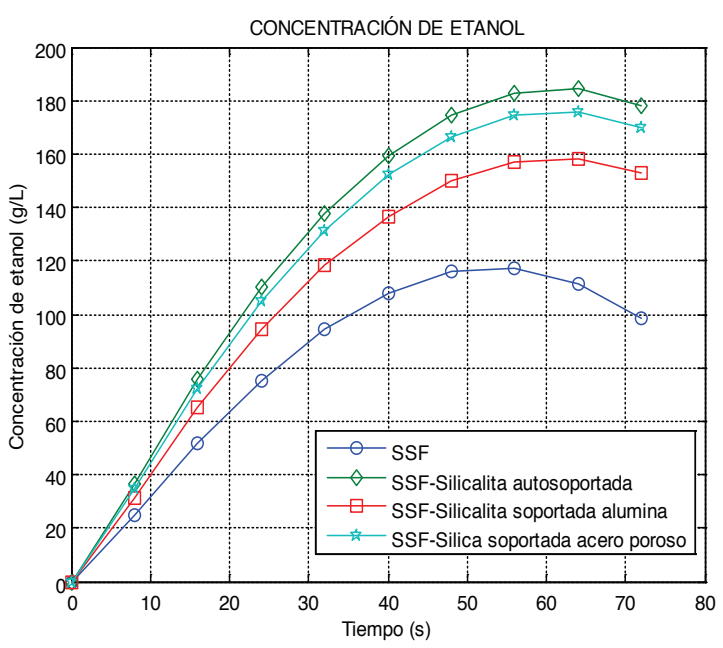

La Fig. 8 muestra los resultados de la simulación de la SSF acoplada a membranas de PDMS y silicalita preparadas sobre diferentes soportes (autosoportadas -este es un caso ideal, pues debido al pequeño espesor de la membrana es necesario tener un soporte-; material polimérico-politetrafluoroetileno-,y acero poroso). El eje $y$ de ambas gráficas representa la concentración de etanol en el permeado a un tiempo dado, mientras que la línea base (curva "SSF") indica la concentración esperada dentro del biorreactor a ese mismo tiempo.

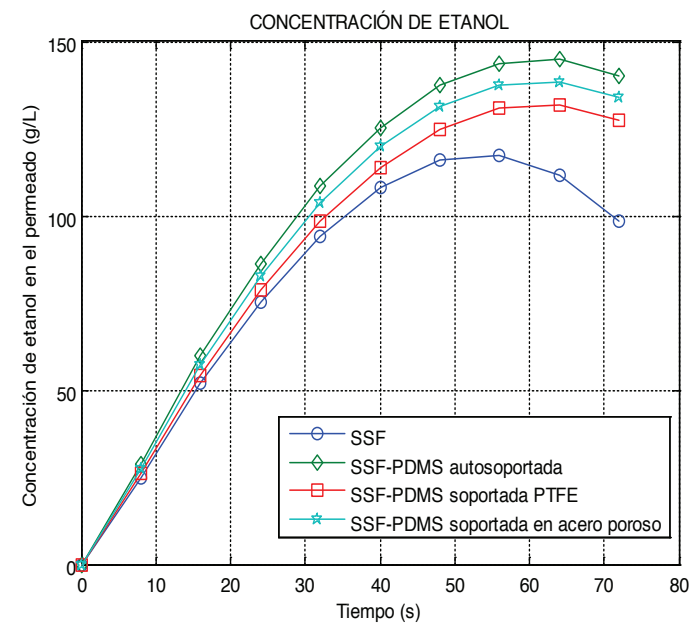


Modelación y simulación de un pervaporador acoplado a un proceso de sacarificación-fermentación para la producción de etanol

Fig. 8. Simulación de la SSF acoplada a membranas de PDMS (izq.) y silicalita (der.). Condiciones de la simulación: temperatura, $35^{\circ} \mathrm{C} ; \mathrm{pH}, 4.5$; concentración enzima, $2 \mathrm{~mL} / \mathrm{L}$; concentración inóculo, $1 \mathrm{~g} / \mathrm{L}$; espesor membrana, $100 \mu \mathrm{m}$; área membrana, $100 \mathrm{~cm}^{2}$; flujo alimentación, $30 \mathrm{~mL} / \mathrm{min}$; presión permeado, $5 \mathrm{mmHg}$.

El efecto de la membrana de silicalita sobre la SSF es mucho más significativo que el efecto de la membrana de PDMS, lo cual se explica por su mayor flux; sin embargo, en ambos casos se obtiene un considerable incremento de la concentración de etanol recuperado cuando el proceso se acopla a las membranas. Además, debe notarse que el tiempo para alcanzar la concentración máxima de etanol se retrasa cerca de 15 horas con respecto al proceso sin separación de etanol. De la misma manera, la incorporación de las membranas aumenta la velocidad de producción de etanol, tal como se puede concluir a partir de la pendiente de las curvas de la Figura 8; todo esto apunta a una mejora en la productividad del proceso global. Como ya se ha dicho, la silicalita, pese a su mejor desempeño, no es un candidato viable para el sistema $\mathrm{SSF}+$ membrana, puesto que presenta mayor susceptibilidad al envenenamiento con los subproductos de la fermentación; por esta razón, se ha explorado la preparación de membranas compuestas PDMS-Silicalita, cuya matriz polimérica mejore la durabilidad de la membrana en las condiciones presentes en el biorreactor.

La Fig. 9 compara el desempeño de las membranas compuestas PDMS-Silicalita, por capas (PC) y mezclada(MX). El comportamiento de las membranas mezcladas es intermedio al de las membranas de silicalita y PDMS; además, puede observarse que la membrana compuesta (con una carga del 50\% de silicalita) presenta mejor desempeño.

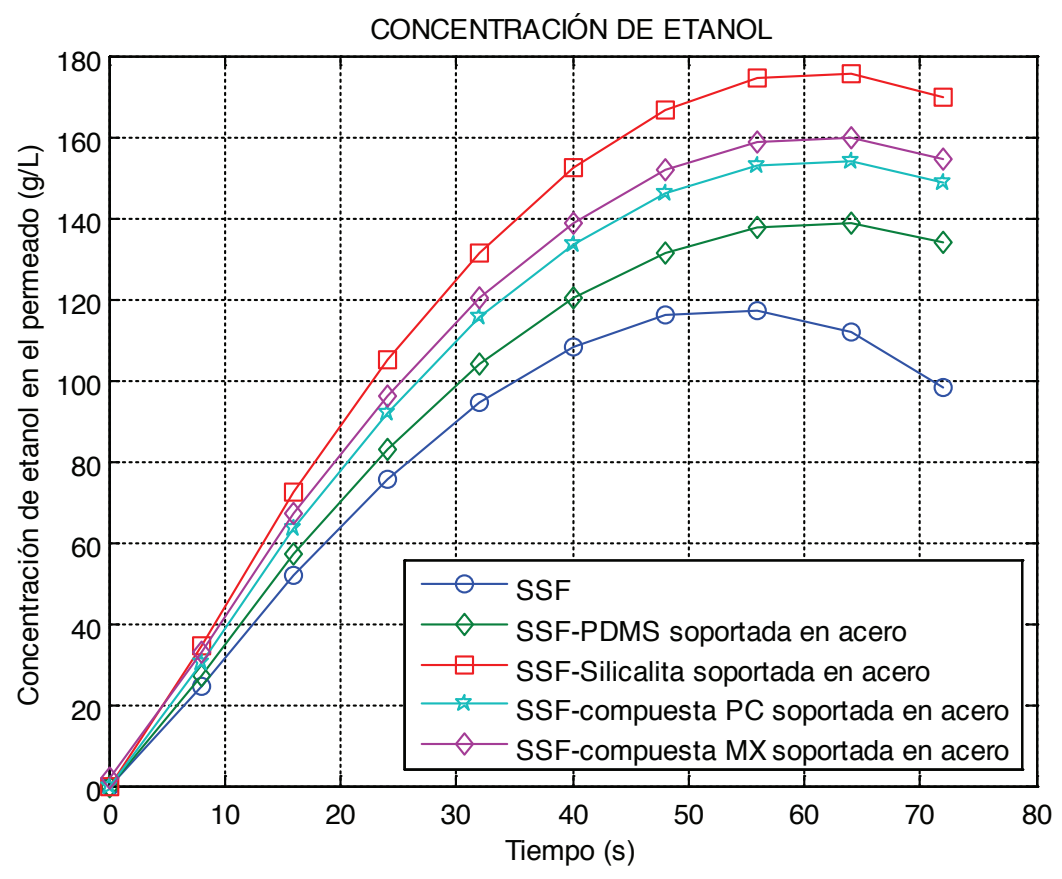

Fig. 9. Simulación de la SSF acoplada a membranas compuestas de PDMS-Silicalita. Condiciones de la simulación: temperatura, $35^{\circ} \mathrm{C} ; \mathrm{pH}, 4.5$; concentración enzima, $2 \mathrm{~mL} / \mathrm{L}$; concentración inóculo, $1 \mathrm{~g} / \mathrm{L}$; espesor membrana, $100 \mu \mathrm{m}$; área membrana, $100 \mathrm{~cm}^{2}$; flujo alimentación, $30 \mathrm{~mL} / \mathrm{min}$; presión permeado, $5 \mathrm{mmHg}$. 


\section{Conclusiones}

Los modelos fenomenológicos desarrollados permiten simular adecuadamente el proceso de sacarificaciónfermentación simultánea, la recuperación de etanol por pervaporación y el proceso acoplado, y estudiar el desempeño del sistema bajo diferentes condiciones de operación. Además, el uso de una membrana compuesta por capas de PDMS+Silicalita es una opción viable para la remoción in-situ de etanol desde el caldo de fermentación; en efecto, esta configuración tiene mayor resistencia al envenenamiento por los subproductos del caldo de fermentación que las membranas de silicalita.

\section{Agradecimientos}

A la Dirección de Investigaciones (DIN) de la Universidad Pedagógica y Tecnológica de Colombia, por la financiación de este trabajo a través del proyecto SGI 1571.

\section{REFERENCIAS}

[1] www.biocombustibles.es, Consultada junio 2015.

[2] R. Wooley et al., "Process design and costing of bioethanol technology: a tool for determining the status and direction of research and development". Biotechnol. Prog Vol. 15, pp. 794-803, 1999.

[3] J. Mielenz, "Ethanol production from biomass: technology and commercialization status". Current Opinion in Microbiology. Vol. 4, pp. 324-329.2001.

[4] C, Wyman, "Ethanol from lignocellulosic biomass: Technology, economics, and opportunities". Bioresource Technol. Vol. 50, pp. 3-16, 1994.

[5] H. Castaño and C. E. Mejía. "Producción de etanol a partir de almidón de yuca utilizando la estrategia de proceso sacarificaciónfermentación simultáneas (SSF)", Vitae, Vol.15, pp. 251-258, 2008.

[6] C. A. Cardona and Ó. J. Sánchez. "Fuel ethanol production: Process design trends and integration opportunities", Bioresource Technology, Vol. 98, pp. 2415-2457, 2007.
[7] K. Wasewr and V. Pangarkar. "Intensification of Recovery of Ethanol from Fermentation Broth Using Pervaporation: Economic Evaluation". Chemical and Biochemical Engineering Quarterly. Vol. 20,pp. 135-145.2006.

[8] F. Lipnitzki and R. Field, "Pervaporation-based hybrid process: a review of process design, application and economics", J. Membr. Sci. Vol. 153, pp. 183-210.1999.

[9] E. Nagy, B. Bakó and L. Szabó, "A kinetic study of the hydrolysis of maltodextrin by soluble glucoamylase". Starch/starke. Vol. 4, pp.145149. 1992.

[10] G. Zanin and F. Moraes, "Modelling cassava starch saccharification with amyloglucosidase", Applied Biochemistry and Biotechnology.Vol. 57/58, pp. 617-625, 1996.

[11] A. Kroumov, A. Modenes and M. Tait, "Development of new unstructured model for simultaneous saccharification and fermentation of starch to ethanol by recombinant strain". Biochemical Engineering Journal. Vol. 28, pp. 243-255. 2006.

[12] M. Matsamura and J. Hirata, "Continuous Simultaneous Saccharification and Fermentation of Raw Starch in a Membrane Reactor", $J$. Chem. Tech. Biotechnol. Vol. 46, pp. 313326.1989 .

[13] C. South, A. Hogsett and L. Lynd, "Modeling simultaneous saccharification and fermentation of lignocelluloses to ethanol in batch and continuous reactors", Enzyme and Microbial Technology.Vol. 17, pp.797-803.1995.

[14] K. Olofsson, A. Rudolf and G. Liden, "Designing simultaneous saccharification and fermentation for improved xylose conversion by a recombinant strain of Saccharomyces cerevisiae", Journal of Biotechnology. Vol. 134, pp.112-120.2008.

[15] S. Ochoa, A. Yoo, J. Repke, G. Wozny and D. Yang, "Modeling and Parameter Identification of the Simultaneous SaccharificationFermentation Process for Ethanol Production", Biotechnol.Prog. Vol. 23, pp.1454-1462. 2007.

[16] J. S. Vrentasand and J. L. Duda, "Diffusion in Polymer-Solvent Systems. Reexamination of the Free-Volume Theory", J. Polymer Sci., Polymer Phys. Ed. Vol. 15, pp. 403.1977. 
Modelación y simulación de un pervaporador acoplado a un proceso de sacarificación-fermentación para la producción de etanol

[17] D. Schuring, Diffusion in Zeolites: Towards a Microscopic Understanding. Data Library Technische Uni-versiteit Eindhoven. 2002.

[18] R. Krishna and R. Baur, "Analytic solution of the Maxwell-Stefan equations for multicomponente permeation across a zeolite membrane", Chem. Eng. J., Vol. 97, pp. 37-45.2004.

[19] S. Dhaval, Pervaporation of solvent mixtures using polymeric and zeolitic membranes: separation studies and modeling, Thesis Doctor of Philosophy at the University of Kentucky, 2001.

[20] L. Li, Z. Xiao, S. Tan, L. Pu and Z. Zhang, "Composite PDMS membrane with high flux for the separation of organics from water by pervaporation", Journal of Membrane Science, Vol. 243, pp.177-187. 2004.

[21] D. Hofmann, L. Fritz and D. Paul, "Molecular modelling of pervaporation separation of binary mixtures with polymeric membranes", Journal of Membrane Science, Vol. 144, pp. 145 159.1998.

[22] S. Takegami, H. Yamada and S. Tsujii, "Pervaporation of ethanol/water mixtures using novel hydrophobic membranes containing polydimethylsiloxane", Journal of Membrane Science, Vol. 75, pp.93-105.1992.

[23] B. Smitha, D. Suhanya, S. Sridhar and M. Ramakrishna, "Separation of organic-organic mixtures by pervaporation - a review", Journal of Membrane Science, Vol. 241, pp.1-21. 2004.

[24] T. Sano, H. Yanagishita, Y. Kiyozumi, F. Mizukami and K. Haraya, "Separation of ethanol/water mixture by silicalite membrane on pervaporation", Journal of Membrane Science, Vol. 95, pp. 221-228, 1994.

[25] M. Nomura, T. Yamaguchi and S. Nakao, "Ethanol/water transport through silicalite membranes", Journal of Membrane Science, Vol. 144, pp.161-171.1998.

[26] T. C. Bowen, R. D. Noble and J. L. Falconer, "Fundamentals and applications of pervaporation through zeolite membranes", Journal of Membrane Science, Vol. 245, pp. 1-33. 2004.

[27] S. L. Wee, C. T. Tye and S. Bhatia, "Membrane separation process-Pervaporation through zeolite membrane", Separation and Purification Technology, Vol. 63, pp. 500-516. 2008.

[28] P. Izak, L. Bartovska, K. Friess, M. Sipek and P. Uchytil, "Description of binary liquid mixtures transport through non-porous membrane by modified Maxwell-Stefan equations", $J$. Membr. Sci. Vol. 214, pp. 293-309.2003.

[29] R. Rautenbach, C. Herion and U. MeyerBlumentoth, "Pervaporation membrane separation processes", R.Y.M. Huang (Ed.), Membrane Science and Technology Series, Vol. 1, Chapter 3, pp. 181-191.1990.

[30] A. Salem, A. A. Ghoreyshi and M. Jahanshahi, "A multicomponent transport model for dehydration of organic vapors by zeolite membranes", Desalination. Vol.193, pp. 35-42. 2006. 